\title{
Determinants of nurses' knowledge gap on pain management in Ghana
}

\author{
Lydia Aziatoa Oluyinka Adejumob \\ a School of Nursing, University of Ghana, P.O. Box LG43, Legon, Ghana \\ b School of Nursing, University of the Western Cape, Private Bag X17, Bellville 7535, South Africa
}

\begin{abstract}
There are concerns about adequacy of nurses' knowledge and skill in effective pain management since effective pain management promotes early recovery after surgery. This study explores factors that accounted for Ghanaian nurses' inadequate knowledge of postoperative pain management using a focused ethnographic design for data collection at a tertiary teaching hospital in Ghana. Fourteen nurses designated as key informants with different backgrounds as nurse educators and leaders were purposively sampled to participate. Data were collected through in-depth individual interviews; all interviews were conducted in English, audio-taped and transcribed verbatim. The study revealed that nurses' inadequate pain management knowledge might have resulted from curriculum gaps during training; inadequate clinical supervision, study days, and workshops for practising nurses; lack of funding for organising regular workshops; and, negative attitudes of nurses whereby new information learned at workshops was not readily applied in clinical practice. It was concluded that nursing curricula at all levels of training in Ghana should incorporate creditbearing courses on pain management, and appropriate pain management education programmes should be instituted for practising nurses. Regular monitoring and evaluation of the impact of such education programs is required.
\end{abstract}

Key words: Pain management, curriculum review, ethnography, qualitative research

\section{INTRODUCTION}

Nursing education has evolved to meet the changing demands of health care and technological advancement. Within the postoperative milieu, the advanced practice nurse with specialist knowledge in pain management offers effective pain control services to patients (Sawhney \& Sawyer, 2008). Previous research has demonstrated that when nurses are knowledgeable about pain management, it results in effective pain control (Zhang et al., 2008). Pain education for nursing students and continuing education for practising nurses 
is deemed necessary to enhance pain management. Structured pain education programmes for nursing students (Watt-Watson et al., 2004) and practising nurses (Francke et al., 1997) have led to improved pain management practices.

Within the surgical context postoperative patients in many countries including Ghana continue to experience moderate to severe pain after surgery (CleggLamptey \& Hodasi, 2005; Qu et al., 2008). Studies have reported that nurses underestimate patients' pain, do not believe patients are in pain, and do not administer the prescribed dosage of analgesics due to fear of addiction (Aziato \& Adejumo, 2013; Rejeh et al., 2009). However, ineffective postoperative pain management poses problems for patients such as pulmonary complications that retard their recovery (Pasero \& McCaffery, 2011).

Consequently, the patient remains admitted for longer and the hospital is not able to admit other patients due to lack of bed-space. The patient is not able to return to work and the family may be adversely affected. The nation may also lose revenue due to the patient's inability to work (McCaffery \& Pasero, 1999).

Nurses caring for surgical patients need expertise in pain management and the use of advanced techniques of pain management such as patient-controlled analgesia, epidural analgesia, and various contemporary pharmacological and non-pharmacological measures used for postoperative pain management (Pasero \& McCaffery, 2011). It is noted, however, that in Ghana, there are no advanced specialist nurses for pain management. Also, advanced techniques requiring contemporary gadgets such as patient-controlled analgesia are not routinely employed in clinical practice.

Previous authors have employed quantitative designs to demonstrate the effect of enhanced knowledge on pain management (Mac Lellan, 2004; Zhang et al., 2008). However, little research employing ethnographic principles has been done to investigate factors that contribute to inadequate knowledge among nurses. Ethnography fosters understanding of the local context or culture that facilitates application of findings from the clinical setting to the context of the study (Hammersley \& Atkinson, 2007).

A type of ethnography applied in nursing research (focused ethnography) explores specific context, and the approach enhances applicability of findings within a particular local environment (Cruz \& Higginbottom, 2013).

A review of the key features of ethnography indicates that the methodology employs the naturalistic model which emphasises that the participants' world should be represented faithfully and data should be collected from their natural environment. One common data collection method used in ethnography is observation also known as naturalistic observation where researchers observe participants in their 'natural' environment. It is noted that researchers should 
analyse the most basic interactions they observe and not just the obvious (O'Reilly, 2005; Saks \& Allsop, 2007; Silverman, 2001).

A recent doctoral study from which this study was derived employed observation to explore issues regarding postoperative pain management within the local context. This paper is derived from individual interviews conducted to gain further understanding of this phenomenon.

From a doctoral study in Ghana it became evident that postoperative pain management strategies and attitudes adopted by surgical nurses indicated inadequate knowledge of postoperative pain management (Aziato \& Adejumo, 2013). This study therefore aimed to explore factors that influenced nurses' inadequate knowledge of postoperative pain management.

\section{METHODOLOGY}

\section{Design}

The study employed a focused ethnographic design that sought to gain a full understanding of the adequacy of the knowledge of postoperative pain management among nurses on a surgical ward. Ethnography is a qualitative methodology that has its' roots in anthropology, and is considered the oldest qualitative methodology.

Nurses started employing ethnographic methodology around the 1960s to learn and have a holistic contextual understanding of nursing phenomena (Buller \& Butterworth, 2001; Oliffe, 2005; Roper \& Shapira, 2000). Nurse researchers over the years have recognized the usefulness of the methodology as it has helped them to understand the meaning of patients' experiences and subsequently develop better ways of providing patient care.

Several nurse researchers have employed the ethnographic methodology to investigate healthcare and nursing issues such as breastfeeding, labour, human immune deficiency virus infection and communication (Arber, 2007; Burnard \& Naiyapatana, 2004; Cricco-Lizza, 2007; Dykes, 2005; El-Nemer et al., 2006). Ethnography has also been employed to investigate postoperative pain assessment (Clabo, 2008).

The ethnographic methodology employs multiple methods of data collection, including interviews. The ethnographic interview involves various respondents, including key informants, based on the research question and the phenomenon under investigation. In ethnography key informants are considered as individuals with special insight or unique experiences about the phenomenon under study. 
Thus the key informant interview may be the primary or sole data collection method, or it may be used in conjunction with other data collection methods (Hammersley \& Atkinson, 2007). This study employed key informant interviews to identify the factors that accounted for inadequate knowledge of postoperative pain management among nurses.

\section{Setting}

The study was conducted at a tertiary teaching hospital (Korle-Bu Teaching Hospital $[\mathrm{KBTH}])$ in Accra. Participants were recruited from the hospital's Continuing Education Unit, Department of Surgery and nursing training institutions based there (Nursing and Midwifery Training School, and the Perioperative and Critical Care Nursing Schools).

\section{Population and sampling}

The study purposively targeted nurses with insight into nursing education especially nurses on the surgical ward and included a nurse leader (1), inservice trainers (3), nursing educators (5), retired nurses (2), and clinical nurses (3). Data saturation from the interviews was achieved after14 participants. Participants were sampled purposively and conveniently according to emerging themes to fully understand issues of pain management education. They were identified as key informants based on their insight into and involvement with education of nurses in the study setting.

The participants were all Ghanaian nurses and aged between 25 and 65 years. They were all Christians and from different ethnic backgrounds in Ghana, such as Ga, Ewe, Akan, and Fante. Participants who were nursing tutors had taught for 2 - 10 years, and those who provided continuing education for nurses had worked as trainers for $3-12$ years.

Clinical nurses had worked on the surgical ward for 2 - 6 years, and the head of nursing had 2 years' experience in that role. The retired nurses had been retired for 2 - 10 years. All of the participants could speak English fluently.

\section{Data collection}

Informed consent was obtained and individual face-to-face interviews were scheduled according to key informants' convenience. Two key informants rescheduled their interviews three times. Interviews were conducted at key informants' offices and an assigned office at the study site. All key informants preferred to be interviewed in English. Interviews focused on education of nurses about pain management, and probes were used to obtain further clarification of respondents' comments. For example, open-ended questions included 'Please tell me about your curriculum content on pain management' and 'Please can you share with me how you run your in-service training programmes?' 
All interviews were recorded with permission from the participants, and lasted for $30-60$ minutes. The first author, who is experienced in interviewing for qualitative studies, conducted all of the interviews. The study ensured that Ghanaian culturally appropriate courtesies were observed during recruitment and interviews such as 'please', 'thank you', appropriate titles for greetings, and observation of bureaucratic protocols. These ensured that detailed information was obtained during the study as participants openly shared their opinions. The observance of the chain of command or bureaucracy ensured that participants had the required institutional backing or authority to share information freely. For example, permission was obtained from the hospital authorities prior to individual informed consent. In addition to interviews, detailed field notes were kept.

\section{Data analysis}

Data analysis occurred concurrently with ongoing interviewing. Interview transcriptions were read several times to obtain full understanding of participants' accounts. The principles of content analysis were employed to develop themes that emerged from the data. The authors discussed interpretations from the data to ensure that themes were fully developed. This process is congruent with ethnographic data analysis (LeCompte \& Schensul, 1999; Roper \& Shapira, 2000). Data were exported into NVivo software, which was subsequently used to manage them. Segments of data that best suited the themes identified were sorted appropriately and used to support findings.

\section{Rigour and ethics}

Ethical clearance for the study was obtained from the ethics committees of the University of the Western Cape and the Ghana Health Service Ethics Committee. Participants were given an information sheet and asked to sign the consent form. They were assured of anonymity and confidentiality.

The study ensured that data were presented in such a way that comments could not be linked to specific key informants. Identification (ID) codes were assigned to participants, such as HN (head of nursing); NT1 - NT3 (in-service trainers); TP (pharmacology tutor); TS1 and TS2 (surgery tutors); DN1 - DN3 (clinical nurses); SP1 and SP2 (peri-operative and critical care tutors); CNLR (retired clinical nurse); and RNE (retired nurse educator). The ID codes were used to present findings.

Rigour was ensured by observing key principles of qualitative interviewing consistent with ethnography, such as, follow-up on participants' comments, and asking for confirmation of understanding derived from comments (Roper \& Shapira, 2000). Transcripts and emerging themes were shared with participants to ensure that comments had been captured accurately (memberchecking). Detailed field notes were written up during data collection and 
analysis to ensure auditability of the study (O'Reilly, 2005; Silverman, 2001). Prolonged field work also ensured that all relevant key informants were interviewed and emerging themes such as curriculum deficit on pain management were fully investigated.

\section{FINDINGS}

Nurses' pre-service education, access to in-service training, and attitudes emerged as important themes in the data. Each theme, including sub-themes, is presented together with accompanying quotes from the data.

\section{Nurses' pre-service education}

This theme describes findings related to pain management knowledge acquisition during training and continuing education on pain management for practising nurses. Two sub-themes were identified: curriculum gap and inadequate clinical teaching.

\section{Curriculum gap}

Participants reported that there were gaps in the curriculum at all levels of training of nurses in Ghana with regard to pain management. There was no separate credit-bearing course on pain management within the curricula for nursing programmes. Pain management was an integral component of courses on other subjects, such as pharmacology and surgical nursing. Participants reported that the time allotted in such courses where pain medication was taught was inadequate to give detailed attention to particular analgesics:

'Pain management is not a course on its own at all levels of training of nurses in this country; it is taught as part of other courses.'(SP1)

'The time for the course is woefully not enough. Just look at the course content; nobody can finish this in one semester; so what happens is that we do the best we can; ... in the second semester when they have filled in the time table and there is a free slot, we use it to top-up and the students do not earn any extra credit for it.' (TP)

\section{Inadequate clinical teaching}

It was reported that student nurses did not receive adequate clinical supervision regarding drug administration as tutors supervise students in all aspects of patient care. Nurse educators felt that some student nurses did not have the requisite clinical environment to put the theoretical knowledge gained in class into practice. The perception that experienced nurses did not allow the student nurse to apply some new techniques in patient care was reported.

'Personally, I have not come to a situation where I have to look at the student administer[ing] pethidine to a patient in pain; because usually when you go 
on clinical supervision it is not just in terms of medication. Although I teach pharmacology,; we do everything.' (TP)

'...the problem our students are facing is about putting into practice what we have taught them. I had an encounter where the qualified nurse said that "she doesn't need a small girl to teach her what to do". ...they are not allowed to practice what they have been taught. If we are able to check that, then we can move forward."'(TS1)

\section{Nurses' in-service training/continuing education}

This theme describes factors that influenced practising nurses' training on specific areas such as pain management. It was realised that practising nurses on the surgical ward did not receive study days or mandatory training programmes. In-service trainers reported training nurses and other health professionals on other equally important health issues such as infection prevention. Sub-themes were lack of study days, workshop participant mix, and budgetary restrictions.

\section{Lack of study days}

The study revealed that some nurses did not have the opportunity to attend workshops regularly to update their knowledge on nursing issues such as pain management. Some nurses did not attend any training programmes in a 12 month period. It was reported that there was no systematic process to ensure that each nurse is trained, but rather that those who showed interest got the opportunity:

'I haven't been to any workshop or conference this year [2012].'(DN3)

'I attend workshops. Attending workshops depends on how you make yourself available, and how serious you are to learn. So, if you are not the type that cares to know what goes on, nobody will be motivated to let you go.'(DN1)

\section{Workshop participant mix}

It was also realised that the surgical unit did not have a training unit to take care of the training needs of nurses working on the surgical ward. Therefore these nurses join other specialty nurses and health professionals for various training programmes organised by the general in-service training unit of the hospital. This training unit does not focus only on postoperative pain management due to the different backgrounds of participants at a particular workshop: 
'...initially when we organise a workshop, we were only concentrating on the nurses; but now we involve all the staff within the hospital. So all the categories of staff that we have such as the $x$-ray, the lab, and environmental staff, records - everybody is on board and we pick nurses not only from the surgical ward but all over. (NT2)

\section{Budgetary restrictions}

It was reported that there were inadequate funds to organise regular workshops for nurses. The lack of funds delayed workshops; sometimes some of the workshops that were scheduled were not held and sometimes the number of participants was reduced.

'...the problem is that every year we have activities planned for the whole year but most of the time, because workshops have to go with money, when our budgets are sent to management they are not approved early. Sometimes they approve only a few workshops. ...because of late approval of our budgets we don't start our workshops early and we do not have the funds to operate on our own.' (NT1)

It was reported that sometimes the surgical nurses were sponsored by the surgical department to attend relevant workshops on subjects such as pain management organised by other organisations. Individual nurses who can afford it also attend these workshops:

'We budget for training needs of nurses such as seminars, workshops, and other courses. So when there is a workshop or seminar, then I decide the number of nurses depending on my budget and sponsor nurses accordingly ... a few nurses sometimes go on their own.'(HN)

\section{Negative attitudes}

It was reported that some nurses did not apply what was learned at workshops on the ward. It was also perceived that some nurses attended the workshop so that they could use the certificate to renew their professional identification number (PIN). It was reported that there was inadequate monitoring and evaluation of the impact of workshops. Nurses were perceived as holding on to previous practices even after a new approach had been taught at workshops:

'...after the workshop, what I realised was that most of them came because of the certificate and they wanted to use it to renew their PIN; so they were not using what they have been taught to work on the ward.' (TS1)

'Attitude is a problem because we realised that there are certain things the nurses don't want to change ... sometimes when you are going to the ward, it's like you are coming as a spy; immediately you are entering the ward, they try to put things right- but what happens when you leave there? You must make 
sure that at every time, things are done correctly. If you put that in mind, you always do your activities right at any moment' (NT1).

\section{DISCUSSION}

The literature has identified inadequate knowledge on pain management among nurses in many countries (Abdalrahim et al., 2011; Wang \& Tsai, 2010). There are indications that nurses' knowledge of pain management is influenced by multifaceted factors that start from pre-service education. In this regard, it has been shown that special pain management education programmes for student nurses lead to improvement in knowledge of and attitudes to pain management.

The authors suggest that the training curriculum should be reviewed to include more content on pain management (Chiang et al., 2006). In this study it was realised, as in other countries (Watt-Watson et al., 2004) that there was inadequate curriculum content on pain management.

This finding presupposes that there is a need for curriculum review to introduce a pain management credit-bearing course (s) in all training programmes in Ghana especially the peri-operative and critical nursing programmes that train nurses specifically for the surgical environment.

There should be well-designed clinical supervision and mentorship systems to ensure that students gain the requisite clinical skills for effective pain management. The importance of highlighting pain education for student nurses has been emphasised by previous authors (Watt-Watson et al., 2004).

Over the years update workshops are organised to enhance nurses' knowledge of pain management. Organisers of such workshops should plan the training programmes carefully, as nurses have reported inadequate information on pain management during training in other countries (Van Niekerk \& Martin, 2001). In this study training of practising nurses to update their knowledge on pain management was inadequate due to a lack of funds to organise workshops. In a resource-limited clinical environment, it is necessary for reprioritisation of resource allocation to focus on enhancing nurses' knowledge of pain management.

It is realised that ineffective postoperative pain management has deleterious effects on the patient and may prolong hospitalisation (Pasero \& McCaffery, 2011). The long-term benefit of effective pain management could also increase revenue for the hospital, as patients may be discharged earlier (MacLaren \& Cohen, 2005). Perhaps workshops could be organised for specialties to ensure depth of information and this could increase applicability of the knowledge gained. Information and training provided in such training sessions should be 
relevant to the resources available to the nurses. The enhanced knowledge could empower practising nurses to teach student and novice nurses to acquire effective pain management skills, as shown in other studies (Sawhney \& Sawyer, 2008; Williamson-Swift, 2007).

Also, the training could improve pain management through effective assessment and reporting (Courtenay \& Carey, 2008). The development and implementation of an effective monitoring and evaluation of patient care system, including pain management, would ensure that knowledge gained at workshops and other up-date courses are applied in practice. Also, specialist programmes at Master's level for advanced practice nurses could prepare nurses for pain management. It has been realised that nurses with specialist training have more knowledge than those trained for general practice (Wilson, 2007).

A previous study in Jordan reported nurses' inadequate knowledge of postoperative pain management. Knowledge was improved after a pain education programme was instituted (Abdalrahim et al., 2011). Also, many studies in other countries have demonstrated that effective education of nurses enhances pain management (Lin et al., 2008; Zhang et al., 2008). In some countries, such as Canada, the United States of America and the United Kingdom, there are specialised pain management nurses that ensure effective pain management in their institutions (Sawhney \& Sawyer, 2008; WilliamsonSwift, 2007).

This study points to the need for nurses to be given study days and provided with the opportunity to undergo some mandatory or core courses based on area of practice. Such regular study days, where nurses attend courses, could provide a structured environment for knowledge acquisition. It would also provide an avenue for nurses to be selective in the courses they attend in order to resolve their knowledge deficits. Such regular courses could be more effective and translate into care outcomes such as effective postoperative pain management and positive attitudes towards pain management (Lin et al., 2008).

Consequently, the need to obtain certificates for renewal of the PIN would not be a driving force for workshop attendance, as is currently the case, as intimated in this study. It is emphasised that the core rationale for up-date courses for nurses is the improvement of patient care outcomes (Courtenay \& Carey, 2008). Hence, improvement of patients' pain management outcomes would indicate the positive impact of nurses' knowledge enhancement programmes in this area (de Rond et al., 2000).

Policy developers and nursing educators should be cautious in applying the findings of the study and its implications among different contexts. Exploration could be undertaken to understand specific local factors within hospitals (Adami \& Kiger, 2005). Further research is needed to employ a quantitative

$$
\text { https://repository.uwc.ac.za/ }
$$


approach to investigate specific areas of knowledge gap among nurses. Also, an intervention study could investigate the effect of specific education programmes on nurses' knowledge and nursing practice.

A limitation of this study could be the small sample and the methodology, making the generalisation of findings in Ghana questionable. However, the intention of qualitative studies is not generalisation of findings but rather the provision of useful insight into a context or phenomenon (Patton, 2002)

\section{CONCLUSION}

The ethnographic approach unearthed contextual factors that contributed to nurses' knowledge gap regarding postoperative pain management. The findings suggested that a programme to enhance postoperative pain management should be holistic and expansive.

It should address the problem at the pre-service level, where curriculum review is required to enhance students' knowledge. Relevant stakeholders should be involved at this level, and nurse educators could be given further training on pain management to ensure that the pain management courses introduced are properly taught. Likewise, regular training for practising nurses should be accompanied by effective monitoring and evaluation of patient care outcomes so that nurses apply in practice what they learn at training programmes.

\section{REFERENCES}

Abdalrahim, M.S., Majali, S.A., Stomberg, M.W., Bergbom, I., 2011. The effect of postoperative pain management program on improving nurses' knowledge and attitudes toward pain. Nurse Education in Practice, 11(4), 250-255.

Adami, M.F., Kiger, A., 2005. A study of continuing nurse education in Malta: the importance of national context. Nurse Education Today, 25(1), 78-84.

Arber, A., 2007. "Pain talk" in hospice and palliative care team meetings: ethnography. International Journal of Nursing Studies, 44(6), 916-926.

Aziato, L., Adejumo, O., 2013. The Ghanaian surgical nurse and postoperative pain management: A clinical ethnographic insight. Pain Management Nursing doi: 10.1016/j.pmn.2012.10.002

.Buller, S., Butterworth, T., 2001. Skilled nursing practice: a qualitative study of the elements of nursing. International Journal of Nursing Studies, 38(4), 405417. 
Burnard, P., Naiyapatana, W., 2004. Culture and communication in Thai nursing: a report of an ethnographic study. International Journal of Nursing Studies, 41(7), 755-765.

Chiang, L.-C., Chen, H.-J. and Huang, L., 2006. Student Nurses' Knowledge, Attitudes, and Self-Efficacy of Children's Pain Management: Evaluation of an Education Program in Taiwan. Journal of Pain and Symptom Management, 32(1), 82-89.

Clabo, L.M.L., 2008. An ethnography of pain assessment and the role of social context on two postoperative units. Journal of Advanced Nursing, 61(5), 531539 .

Clegg-Lamptey, J.N.A., Hodasi, W.M., 2005. An audit of aspects of informed consent and pain relief in general surgical units of Korle-Bu Teaching Hospital. Ghana Medical Journal, 39(2), 63-67.

Courtenay, M. and Carey, N., 2008. The impact and effectiveness of nurse-led care in the management of acute and chronic pain: a review of the literature. Journal of Clinical Nursing, 17(15), 2001-2013.

Cricco-Lizza, R., 2007. Ethnography and the generation of trust in breastfeeding disparities research. Applied Nursing Research, 20(4), 200-204.

Cruz, E.V., Higginbottom, G., 2013. The use of focused ethnography in nursing research. Nurse Researcher, 20(4), 36-43.

de Rond, M.E.J., de Wit, R., van Dam, F.S.A.M., Muller, M.J., 2000. A pain monitoring program for nurses: Effect on the administration of analgesics. Pain, 89(1), 25-38.

Dykes, F., 2005. A critical ethnographic study of encounters between midwives and breast-feeding women in postnatal wards in England. Midwifery, 21(3), 241-252.

El-Nemer, A., Downe, S., Small, N., 2006. 'She would help me from the heart': ethnography of Egyptian women in labour. Social Science \& Medicine, 62, 8192.

Francke, A.L., Luiken, J.B., de Schepper, A.M.E., Huijen Abu-Saad, H., Grypdonck, M., 1997. Effects of a continuing education program on nurses' pain assessment practices. Journal of Pain and Symptom Management, 13(2), 90-97.

Hammersley, M., Atkinson, P., 2007. Ethnography: Principles in Practice. Routledge, London.

LeCompte, M.D., Schensul, J.J., 1999. Analyzing and Interpreting Ethnographic Data: Ethnographer's Toolkit 5. Altamira Press, London. 
Lin, P.-C., Chiang, H.-W., Chiang, T.-T., Chen, C.-S., 2008. Pain management: evaluating the effectiveness of an educational programme for surgical nursing staff. Journal of Clinical Nursing, 17(15), 2032-2041.

Mac Lellan, K., 2004. Postoperative pain: Strategy for improving patient experiences. Journal of Advanced Nursing, 46(2), 179 - 185.

MacLaren, J.E., Cohen, L.L., 2005. Teaching behavioural pain management to healthcare professionals: A systematic review of research in training programs. The Journal of Pain, 6(8), 481-492.

McCaffery, M., Pasero, C., 1999. Pain: Clinical Manual. Mosby Inc, London.

O'Reilly, K., 2005. Ethnographic methods. Routledge Taylor \& Francis Group, London.

Oliffe, J., 2005. Demystifying nursing research. Why not ethnography? Urologic Nursing, 25(5), 395-399.

Pasero, C., McCaffery, M., 2011. Pain Assessment and Pharmacologic Management. Mosby/Elsevier, St. Louis.

Patton, M.Q., 2002. Qualitative Research and Evaluation Methods. Sage Publications Inc., London.

Qu, S., Sherwood, G.D., McNeill, J.A., Zheng, L., 2008. Postoperative pain management outcome in Chinese inpatients. Western Journal of Nursing Research, 30(8), 975-990.

Rejeh, N., Ahmadi, F., Mohammadi, E., Kazemnejad, A., Anoosheh, M., 2009. Nurses' experiences and perceptions of influencing barriers to postoperative pain management. Scandinavian Journal of Caring Sciences, 23(2), 274-281.

Roper, J.M., Shapira, J., 2000. Ethnography in Nursing Research. Sage Publications, Inc, California.

Saks, M. and Allsop, J., 2007. Researching Health: Qualitative, Quantitative and Mixed Methods. Sage, London.

Sawhney, M., Sawyer, J., 2008. A cross-sectional study of the role of Canadian nurses with a specialty practice in pain management. Acute Pain, 10(3-4), 151156.

Silverman, D., 2001. Interpreting Qualitative Data: Methods for Analysing Talk, Text and Interaction. Sage, London.

Van Niekerk, L. M., \& Martin, F. (2001). Tasmanian nurses' knowledge of pain management. International Journal of Nursing Studies, 38(2), 141-152. 
Wang, H.-L. and Tsai, Y.-F., 2010. Nurses' knowledge and barriers regarding pain management in intensive care units. Journal of Clinical Nursing, 19(2122), 3188-3196.

Watt-Watson, J., Hunter, J., Pennefather, P., Librach, L., Raman-Wilms, L., Schreiber, M., et al., 2004. An integrated undergraduate pain curriculum, based on IASP curricula, for six Health Science Faculties. Pain, 11O(1-2), 140-148.

Williamson-Swift, A., 2007. Education and training of pain nurse specialists in the United Kingdom. Acute Pain, 9(4), 207-213.

Wilson, B., 2007. Nurses' knowledge of pain. Journal of Clinical Nursing, 16(6), 1012-1020.

Zhang, C.-H., Hsu, L., Zou, B.-R., Li, J.-F., Wang, H.-Y., Huang, J. (2008). Effects of a Pain Education Program on Nurses' Pain Knowledge, Attitudes and Pain Assessment Practices in China. Journal of Pain and Symptom Management, 36(6), 616-627. 